\title{
Synthesis, antimicrobial activity and structural studies of low molecular mass lysine dendrimers ${ }^{\star}$
}

\author{
Jolanta Janiszewska ${ }^{1}$ and Zofia Urbańczyk-Lipkowska ${ }^{2 凶}$ \\ ${ }^{1}$ Industrial Chemistry Research Institute, Warszawa, ${ }^{2}$ Institute of Organic Chemistry PAS, Warszawa, Poland \\ 凶e-mail: ocryst@icho.edu.pl
}

Received: 06 November, 2005; revised: 19 January, 2006; accepted: 16 February, 2006 available on-line: 23 February, 2006

\begin{abstract}
Four low molecular mass lysine dendrimers were synthesized by Boc chemistry in solution (155 and 169) and Fmoc chemistry on solid support (P2 and P13). The structure and fragmentation mode of the above dendrimers was investigated in gas phase by the LSI-MS and ESI-MS techniques. ${ }^{1} \mathrm{H}$ and ${ }^{13} \mathrm{C}$ NMR analysis in solution ( $d_{6}$-DMSO) allowed to confirm the correct structure. Antimicrobial activities of the dendrimers against Staphylococcus aureus, Escherichia coli and Candida albicans confirmed our hypothesis that the dendrimer structure can be used for construction of molecules interacting with biological membranes.
\end{abstract}

Keywords: antibiotic resistance, natural antibacterial peptides, basic dendrimers, mass spectrometry (MS), nuclear magnetic resonance (NMR).

Dendrimers are a new group of macromolecules characterized by branched architecture. Dendrimers are most often synthesized in an iterative sequence of reaction steps, in which each additional iteration leads to a higher generation molecules. The structural perfection (monodispersion) of dendrimers in comparison with other nanomolecules, i.e. polymers, makes them very attractive novel materials in many areas of technology (Boas \& Heegard, 2004). Their characteristic feature is the possibility of locating a well defined number of active elements at the outer shell. In medicine, they have been postulated as model antigens, anticancer and antiviral agents and drug carriers. Peptide dendrimers constitute a special class that can be generally defined as macromolecules containing peptide bonds in their structure (Cloninger, 2002; Driffield et al., 2003). Among them amino acids functionalized dendrimers (e.g. PAMAM) are the most popular. Dendrimers can also be prepared exclusively from amino acids. In this case, lysine can be used as a nonsymmetrical dior trivalent core and branching unit. Over 20 years of intensive research has resulted in an increasing number of patents and patent applications with the "dendrimer" keyword. This suggests that biomedical applications may appear in near future.

At about the same time when the first papers on the synthesis and applications of dendrimers were published, other interesting macromolecules with antimicrobial properties were discovered. The so called "antimicrobial peptides" have been found in many living organisms and play an important role in their defense systems (Reddy et al., 2004). They have the property of unselective or selective killing of pathogens, including Gram negative and Gram positive bacteria, yeasts and viruses (Ganz 2003; Kupper \& Fuhlbrigge, 2004). Among them a large group - presently about 600 compounds, are qualified as "cationic peptides". Cationic antimicrobial peptides are generally defined as linear peptides of less than 50 amino-acid residues with an overall positive charge, imparted by the presence of multiple lysine and arginine residues, and a substantial portion ( $50 \%$ or more) of hydrophobic/aromatic residues. Some of them are linear whereas others are cyclic due to the existence of one or more disulfide

^Presented at the 18th Polish Peptide Symposium, 4-8 September, 2005, Wrocław, Poland.

Abbreviations: Arg, L-arginine; BA, benzylamine; Boc, tert-butyloxycarbonyl; ESI-MS, electrospray ionization mass spectrometry; Fmoc, fluorenyl-9-methoxycarbonyl; HPLC, high pressure liquid chromatography; LSI-MS liquid secondary ion mass spectrometry, Lys, L-lysine; MIC, minimal inhibitory concentration; Phe, L-phenylanine. 
bridges. Some adopt $\alpha$-helical, $\beta$-sheet or both $\beta$ sheet and $\alpha$-helical structures with the first two classes being the most common in nature. Despite the different folding behavior in the vicinity of biological membranes, most peptides seem to adopt an amphipathic structure with opposing hydrophobic and positively charged faces (Powers \& Hancock, 2003; Papo \& Shai, 2003; Lequin et al., 2003). Structure-activity studies of these peptides reveal two main requirements for the antimicrobial activity, (1) a cationic charge, and (2) an induced amphipathic conformation.

While looking at the structural characteristics of the antimicrobial peptides we came to a conclusion that the repetitive character of their building blocks might be well reproduced by the use of a dendrimeric structure. Therefore, our goal was to synthesize a new class of low molecular mass peptide dendrimers, designed as branched analogs of the natural linear, basic antimicrobial peptides. Both groups contain common elements responsible for interactions with biological membranes, i.e. basic amino acids (lysine, arginine, ornithine) and amino acids containing lypophilic/aromatic residues. As their topological relation is critical for expressing the antimicrobial properties we proposed to locate appropriate residues not only at the surface of the dendrimer but also within the dendrimer tree. The obtained compounds were active against Gram negative (Escherichia coli) and Gram positive (Staphylococcus aureus) bacteria as their natural linear precursors, with the MIC in the range 16-1300 $\mu \mathrm{M} / \mathrm{L}$ (Janiszewska et al., 2003). The present paper presents the synthesis of four lysine dendrimers of the structures shown below. Several structural problems characteristic for this class of compounds will be shown on the basis of mass spectrometry and NMR techniques.

\section{MATERIALS AND METHODS}

All solvents and reactants were of analytical grade and were used without further purification. Mass spectra were recorded with a Mariner ESI time-of-flight mass (PerSeptive Biosystems) and AMD-604 (AMD Intectra GmbH) spectrometers. The needle voltage was adjusted to $5 \mathrm{kV}$; declustering potential was varied from 20 to $220 \mathrm{~V}$. Samples were prepared in methanol. NMR spectra were recorded using a Bruker Avance 500 device in $d_{6}$-DMSO.

Synthesis and assay of antimicrobial activity. The peptides P2 and P13 were synthesized on solid support using Fmoc protected amino acids. Piperidine $(20 \%)$ in DMF was used to remove the Fmoc groups. Dendrimers 155 and 169 were synthesized by the Boc chemistry in solution. The peptides were converted into an amide form by treating with am-
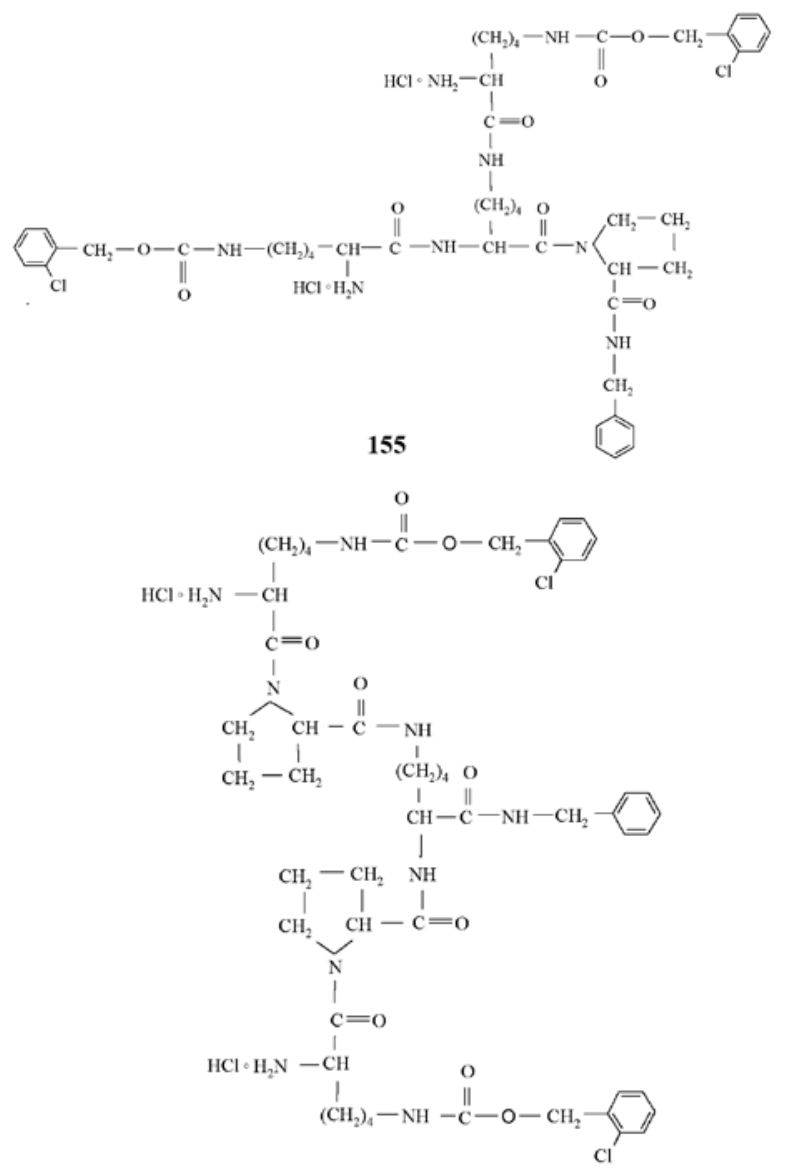

169

monia in methanol overnight. All crude peptide dendrimers were purified by gel filtration on Sephadex LH-20, followed by preparative HPLC. The fraction containing pure peptide was lyophilized twice and purity was confirmed by analytical HPLC and mass spectrometry. Antimicrobial activity was assayed against S. aureus NCTC 4163 and E. coli NCTC 8196. To determine the minimum inhibitory concentration (MIC), the microdilution broth method was used. Antimicrobial activities were expressed as the minimal inhibitory concentration - the concentration at which $100 \%$ inhibition of growth was observed. Three independent experiments were averaged and calculated deviations were less than $15 \%$.

\section{RESULTS AND DISCUSSION}

\section{Synthesis and antimicrobial activity}

The designed dendrimers belong to two dendrimer families. Dendrimer $\mathbf{1 5 5}$ is built from a lysine core functionalized at the C-end by Pro-BA $(\mathrm{BA}=$ benzylamine) and terminated with two $\varepsilon-N-2$-chlorobenzyloxycarbonyl-protected lysines. Dendrimer 169 has BA attached to the C-end and two Pro resi- 

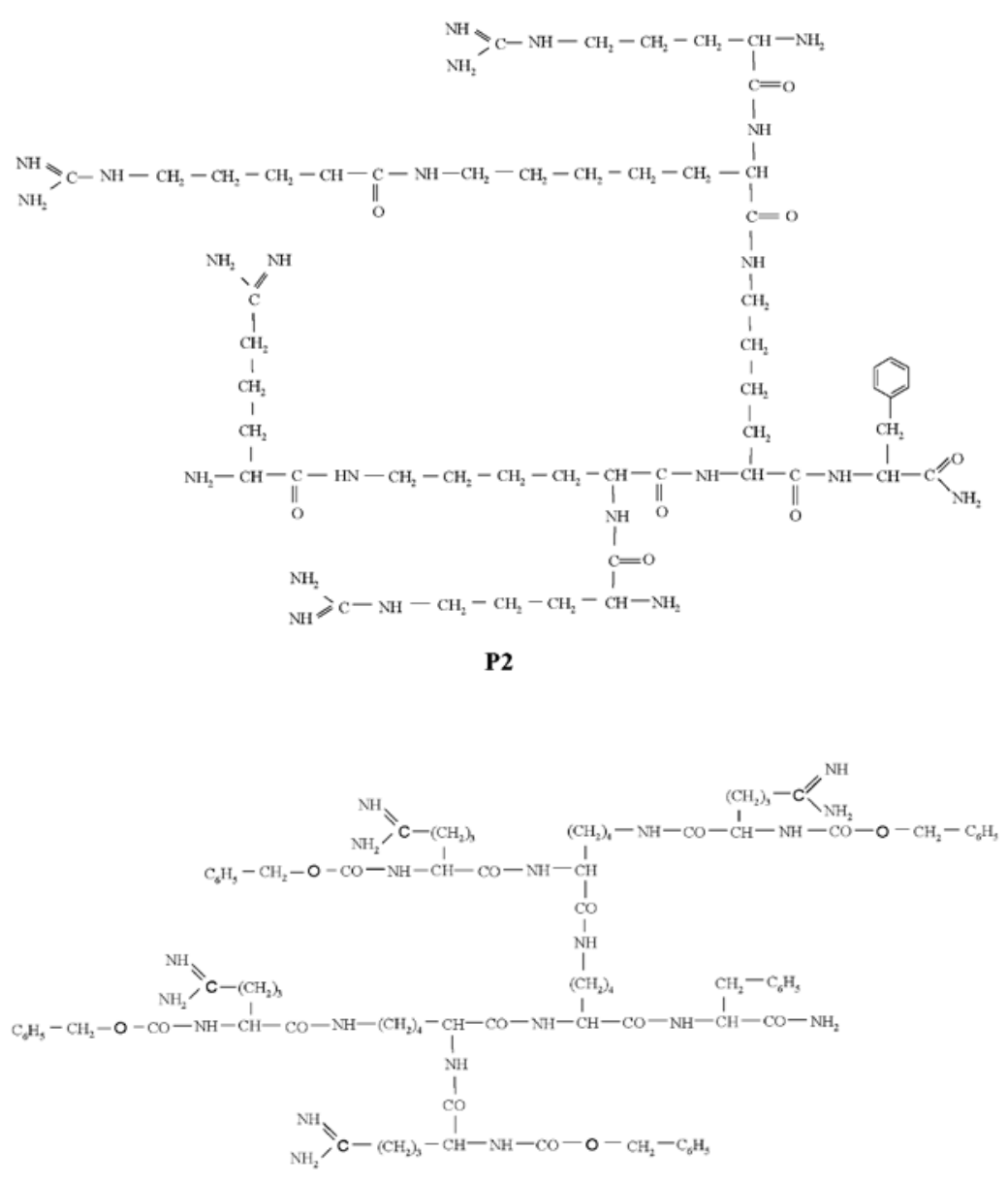

P13

dues that separate two branching lysines from the lysine core. Dendrimers P2 and P13 are lysine dendrimers of the first generation functionalized at the periphery by four Arg residues with free and Bocprotected $\alpha$-amino groups in $\mathbf{P} 2$ and $\mathbf{P 1 3}$, respectively, and with Phe attached to the C-end. According to the published data, the majority of dendrimers containing amino acids were synthesized on solid support. In the present case, dendrimers 155 and

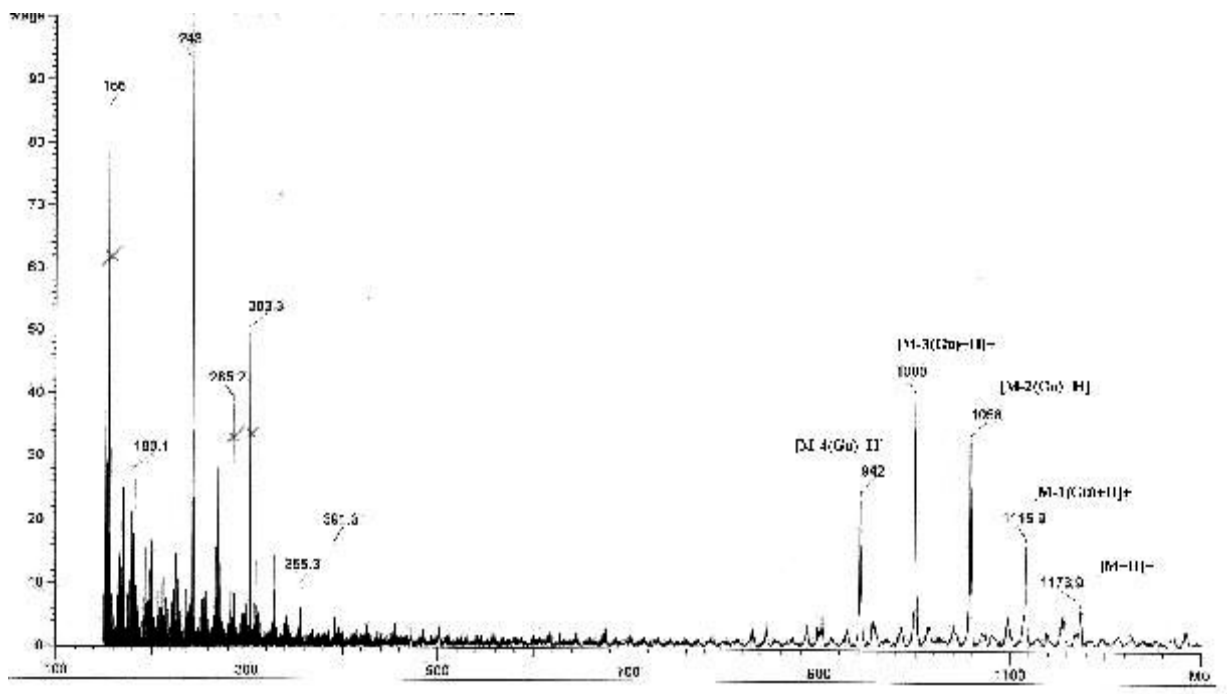

169 were synthesized in solution by DCC coupling, whereas P2 and P13 on a functionalized resin using Fmoc methodology. The synthetic strategy adopted for preparation of peptide dendrimers allowed building subsequent generations of branches with high certainty. Gel filtration on Sephadex LH-20 after each reaction step in the case of the solution method, followed by preparative and analytical HPLC showed single products of high purity (about 80\% yield). The dendrimers synthesized on solid support were obtained in lower yields (about 40\%). In the case of spontaneous dendrimerization, which is a common technique during dendrimers synthesis, structural imperfection is enhanced with growing generations. That includes incomplete substitution and folding back with formation of cyclic structures inside of the dendrimer. Our lysine-based dendrimers have not very dense structure and are homogenous.

The antimicrobial activities of the dendrimers against $S$. aureus NCTC 4163 (Gram-positive), E. coli NCTC 8196 (Gram-negative) and Candida albicans are shown in $\mathrm{Ta}-$ ble 1. It appears that the arginine-containing compounds P2 and P13 are two-fold more selective towards Gram(-) than Gram(+) bacteria. In the second pair of compounds, compound $\mathbf{1 6 9}$ is four times more selective than 155 towards Gram(+) bacteria. That confirms our hypothesis that dendrimer structure can be used for construction of molecules interacting with biological membranes.
Figure 1. LSI-MS spectrum of $\mathrm{P} 2$ in DTE/ DTT matrix.

Bands from the matrix are X-marked. 


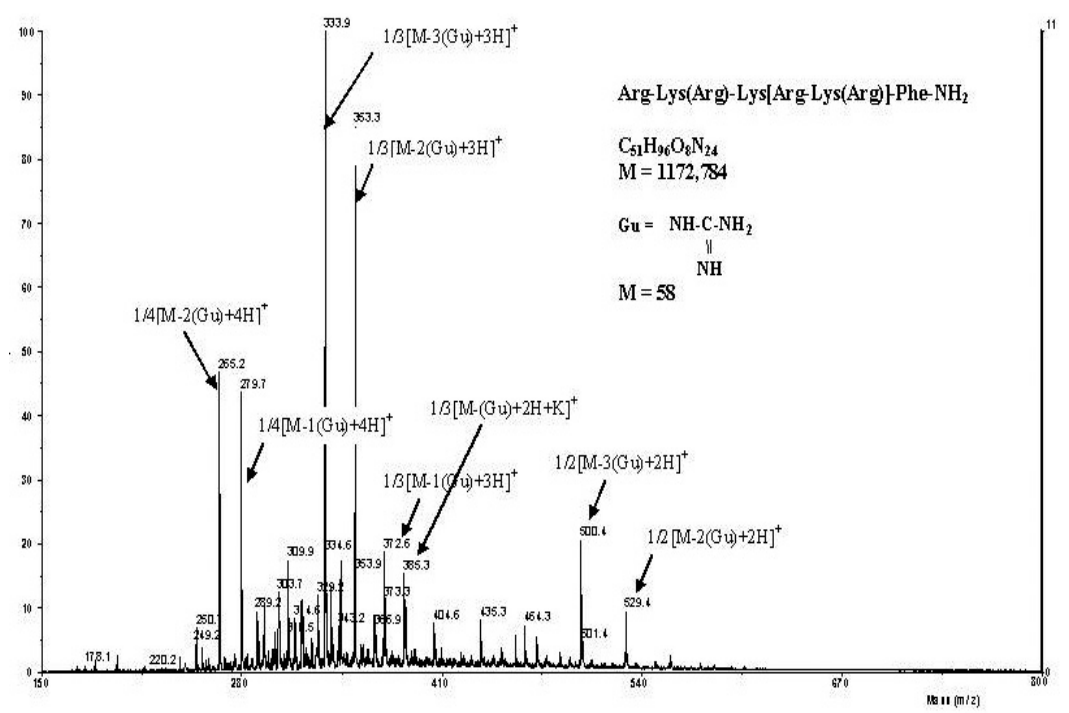

Figure 2. ESI-MS spectrum of $\mathbf{P 2}$.

Sample dissolved in methanol, declustering potential $-100 \mathrm{~V}$.

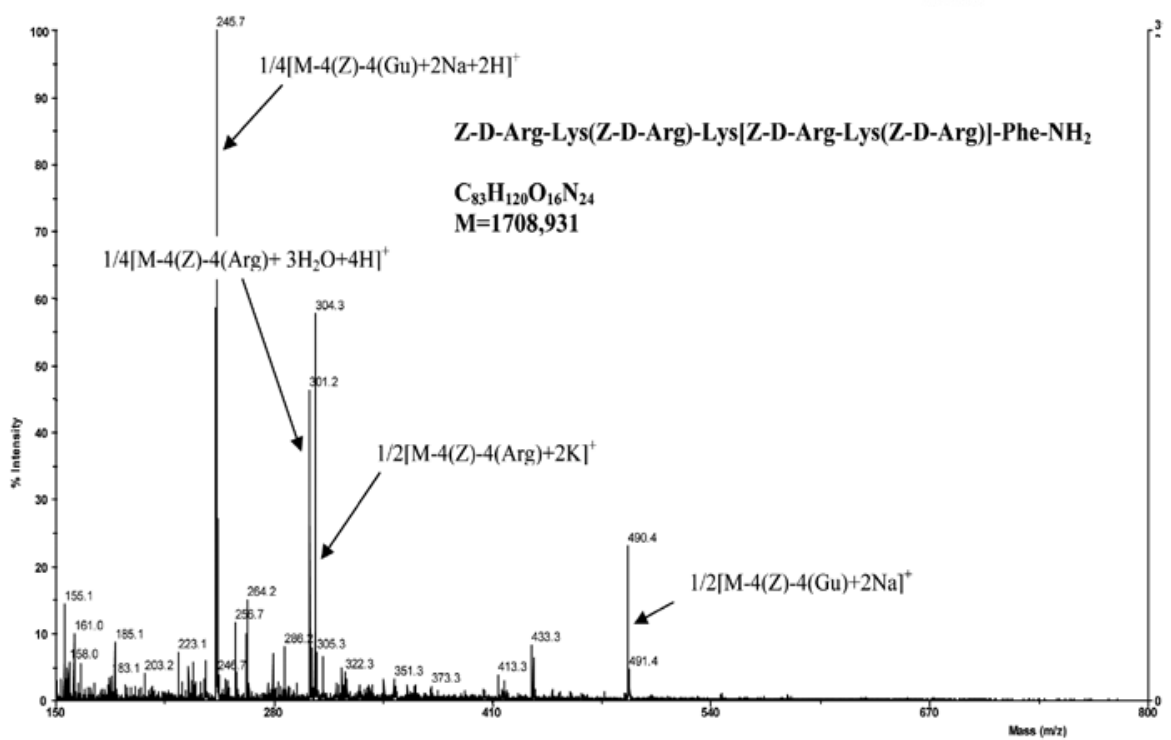

Figure 3. ESI-MS spectrum of P13.

Sample in methanol, declustering potential 110 V.

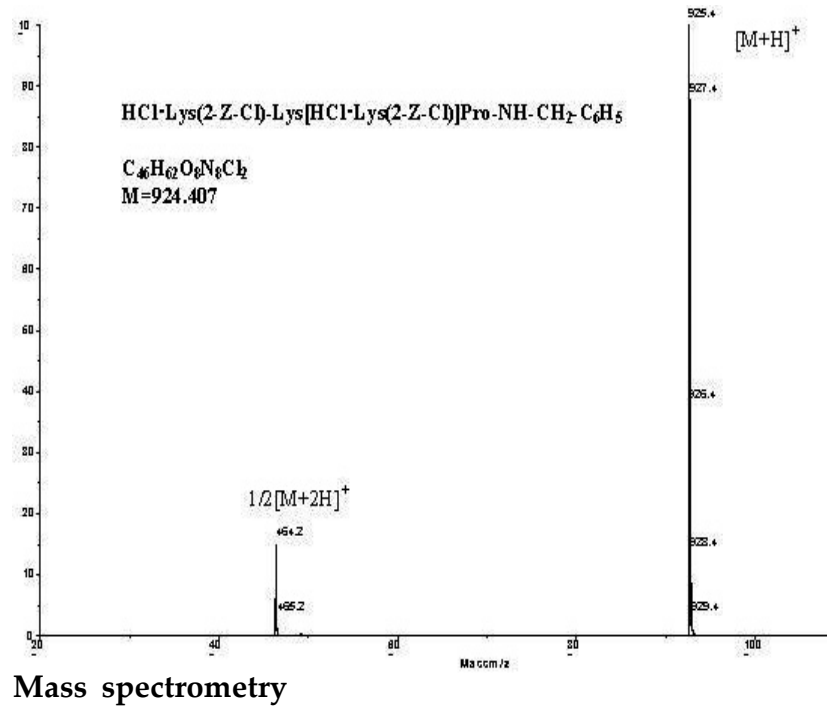

Analytical data for small homogenous dendrimeric peptides are scarce. Therefore, it was of interest to see how a branched structure and presence of several copies of basic amino acids (Lys, Arg)

will affect their mass spectra. The mass spectra were measured in various experimental conditions and analyzed in correlation with their structure. Among the available mass spectrometry methods were: liquid secondary ion mass spectrometry - LSIMS, and electrospray ionization mass spectrometry 
Table 1. Antimicrobial activity of dendrimers

\begin{tabular}{lccc}
\hline Compound & $\begin{array}{c}\text { MIC }(\mu \mathrm{M}) \\
\text { S. aureus }\end{array}$ & $\begin{array}{c}\text { MIC }(\mu \mathrm{M}) \\
\text { E. coli }\end{array}$ & $\begin{array}{c}\text { MIC }(\mu \mathrm{M}) \\
\text { C. albicans }\end{array}$ \\
\hline P2 & 144 & 72 & 72 \\
P13 & 69 & 32 & 69 \\
$\mathbf{1 5 5}$ & 75 & 75 & 75 \\
$\mathbf{1 6 9}$ & 64 & 254 & 254 \\
\hline
\end{tabular}

- ESI-MS. Dendrimers P2 and P13 contain eight and four protonation centers, respectively. Figures 1 and 2 show LSI-MS and ESI-MS spectra of compound P2, containing four unsubstituted arginines at the surface and four amino groups inside of the dendrimer tree. LSI-MS spectrum of $\mathbf{P} 2$ obtained in DTE/DTT matrix (Fig. 1) gave a low-intensity molecular peak $[\mathrm{M}+\mathrm{H}]^{+}$at 1173.9 and four more intensive bands obtained due to cleavage of one, two, three and four guanidine units of $(m=58$ a.u.). The ESI-MS spectrum for positive ion detection mode for the same compound was more complex and showed exclusively multiply charged ions which appeared after cleavage of one to three guanidine units and addition of two to four protons (Fig. 2). Dendrimer P13 containing also four arginines at the periphery of the molecule has a similar fragmentation behavior in ESI experiment (Fig. 3). A typical ESI mass spectrum for dendrimers without Arg is shown in Fig. 4. It contains a molecular peak $[\mathrm{M}+\mathrm{H}]^{+}$and ion $1 / 2[\mathrm{M}+2 \mathrm{H}]^{+}$due to protonation of the second amine group of compound 155 .

In structural studies of dendrimeric compounds mass spectrometry is very often a method of choice, confirming both chemical purity and proper structure (fragmentation). We found that electrospray method of ionization (ESI-MS) has been particularly valuable for our polyionic dendrimeric peptide molecules.

\section{NMR spectroscopy}

The dendrimers 155 and 169 contain numerous polar amide groups and were obtained in the form of highly hygroscopic diammonium salts. Therefore, the samples had been lyophilized for $24 \mathrm{~h}$ at $-40^{\circ} \mathrm{C}$ before NMR experiments were performed. Even with this treatment elementary analysis of compound 155 showed that one water of crystallization was present in the freshly lyophilized sample.

The ${ }^{1} \mathrm{H}$ NMR spectrum of the $\varepsilon-N-2$-chlorobenzyloxycarbonyl-protected first generation dendrimer 155, recorded in $\left(\mathrm{CD}_{3}\right)_{2} \mathrm{SO}$ is shown in Fig. 5. A characteristic feature of this system is that signals for specific protons are located in different spectral regions. The signals of $\mathrm{NH}_{2}$ and $\mathrm{NH}$ groups are located in the range 6.93-8.70, with protons belonging to the two $\mathrm{NH}_{2}$ groups resonating at 8.20 and 8.25, respectively. The highest downshift is observed for the G-0 NH proton of lysine (8.70). The signals for the aromatic protons are located between 7.22-7.44. Characteristic is the region at $\delta=5.1$, where the resonance peaks of the four peripheral benzyl $\mathrm{CH}_{2}$ are localized. The methylene group ${ }^{1} \mathrm{H}$ signals assigned using TOCSY experiment were almost the same for the two terminal G-1 lysines. The signals of the G0 protons of lysine were slightly downfield-shifted with the two $C_{(\beta)}$ protons resonating at 1.56 and 1.71 . The signals of the $\mathrm{C}_{(\alpha)}$ of the three Lys and Pro protons resonate between 3.74 and 4.49. Similarly, the ${ }^{13} \mathrm{C}$ NMR resonance signals of the various functional entities of the polylysine dendrimers were located in distinct spectral regions. Three sets of carbonyl signals could be identified; one originated from the Lys amide group (168.13-169.53) and the two other due to the aromatic protective groups (155.67) and to Pro (171.44).

The basic dendrimeric peptides studied here were prepared from the corresponding $\alpha$ - or $\varepsilon$-Bocprotected derivatives. For the Boc-group deprotec-

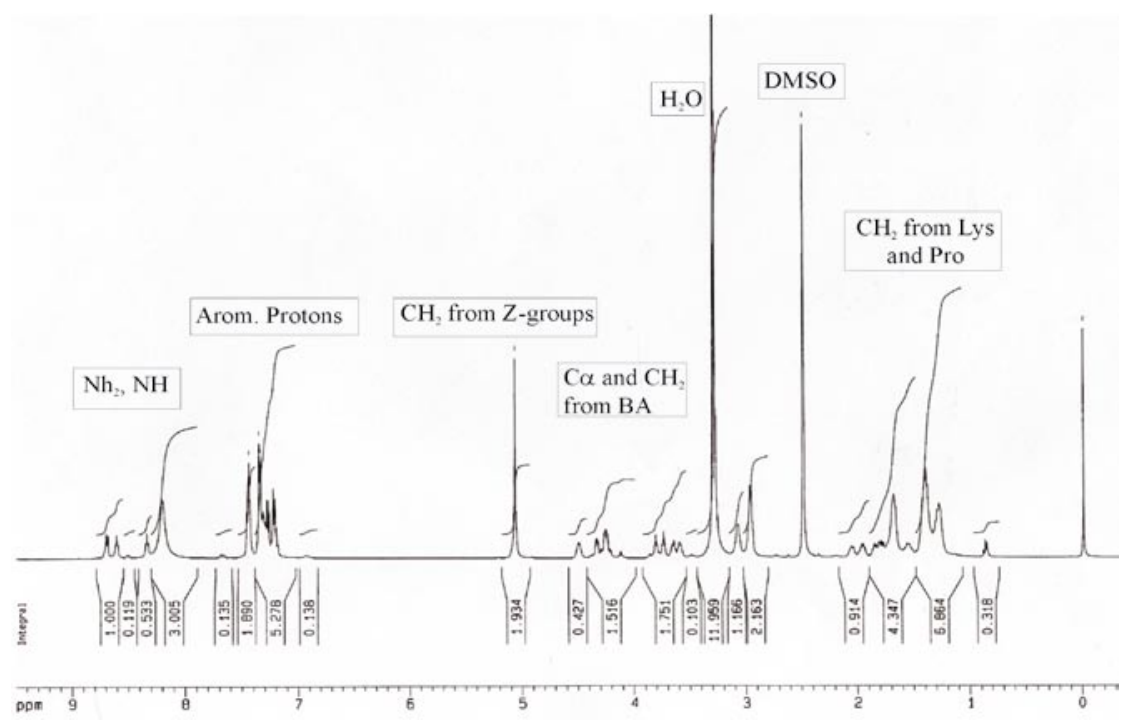

Figure 5. ${ }^{1} \mathrm{H}$ NMR spectrum of dendrimer 155 in $\left(\mathrm{CD}_{3}\right)_{2} \mathrm{SO}$. 
tion hydrochloric acid in ethyl acetate was used. However, ESI-MS spectra of the dendrimer 169, obtained at various declustering potentials suggested that deprotection was not completed. This was confirmed by the the ${ }^{1} \mathrm{H}$ NMR spectrum of 169 , where singlet of the Boc groups methyl protons appeared at 1.4. ${ }^{13} \mathrm{C}$ NMR spectra for this compound contained methyl group signals at 28.8-28.9 and also signals splitting in the $\mathrm{C}=\mathrm{O}$ region. To obtain full Boc group deprotection, $\mathrm{HCl} / \mathrm{AcOH}$ mixture had to be used.

Structural studies of large dendrimers by NMR spectroscopy are time-consuming and complicated due to close contacts of large molecular fragments (the effect of branching) and multiple occurrence of the same sequence of atoms. However, with the use of 2D techniques complete band assignment is possible. NMR spectroscopy showed that in the $\varepsilon-N-2$-chlorobenzyloxycarbony-protected dendrimer 155, the $\mathrm{NH}$ and $\mathrm{CH}_{2}$ signals of the core and the first generation lysines can be differentiated. Similarly, the aliphatic ${ }^{13} \mathrm{C}$ signals due to G-0 and G-1 lysine could also be separated.

\section{Acknowledgements}

This work was supported by the Ministry of Higher Education and Informatics, grant No. 3T09B 11528.

\section{REFERENCES}

Boas U, Heegaard PMH (2004) Dendrimers in drug research. Chem Soc Rev 33: 43-63.

Cloninger MJ (2002) Biological applications of dendrimers. Curr Opin Chem Biol 6: 742-748.
Driffield M, Goodall DM, Smith DK (2003) Syntheses of dendritic branches based on L-lysine: is the stereochemistry preserved throughout the synthesis? Org Biomol Chem 1: 2612-2620.

Frechet JM, Tomalia DA (2002) Dendrimers and other dendritic polymers. Wiley Series in Polymer Science.

Ganz T (2003) Defensins: antimicrobial peptides of innate immunity. Nat Rev Immunol 3: 710-720.

Janiszewska J, Swieton J, Lipkowski AW, Urbanczyk-Lipkowska Z (2003) Low molecular mass peptide dendrimers that express antimicrobial properties. Bioorg Med Chem Lett 13: 3711-3713.

Kupper TS, Fuhlbrigge RC (2004) Immune surveillance in the skin: mechanisms and clinical consequences. Nat Rev Immunol 4: 211-222.

Lequin O, Bruston F, Convert O, Chassaing G, Nicolas P (2003) Helical structure of dermaseptin B2 in a membrane-mimetic environment. Biochemistry 42: 1031110323.

Papo N, Shai Y (2003) Exploring peptide membrane interaction using surface plasmon resonance: differentiation between pore formation versus membrane disruption by lytic peptides. Biochemistry 42: 458-466.

Powers JPS, Hancock RE (2003) The relations between peptide structure and antibacterial activity. Peptides $\mathbf{2 4}$ : 1681-1691.

Reddy KVR, Yedery RD, Aranha C (2004) Antimicrobial peptides: premises and promises. Int J Antimicrobal Agents 24: 536-547. 